\title{
Individual neuronal subtypes control initial myelin sheath growth and stabilization
}

\author{
Heather N. Nelson, Anthony J. Treichel, Erin N. Eggum, Madeline R. Martell, Amanda J. Kaiser, Allie G. Trudel,
} James R. Gronseth, Samantha T. Maas, Silas Bergen and Jacob H. Hines ${ }^{*}$ (i)

\begin{abstract}
Background: In the developing central nervous system, pre-myelinating oligodendrocytes sample candidate nerve axons by extending and retracting process extensions. Some contacts stabilize, leading to the initiation of axon wrapping, nascent myelin sheath formation, concentric wrapping and sheath elongation, and sheath stabilization or pruning by oligodendrocytes. Although axonal signals influence the overall process of myelination, the precise oligodendrocyte behaviors that require signaling from axons are not completely understood. In this study, we investigated whether oligodendrocyte behaviors during the early events of myelination are mediated by an oligodendrocyte-intrinsic myelination program or are over-ridden by axonal factors.
\end{abstract}

Methods: To address this, we utilized in vivo time-lapse imaging in embryonic and larval zebrafish spinal cord during the initial hours and days of axon wrapping and myelination. Transgenic reporter lines marked individual axon subtypes or oligodendrocyte membranes.

Results: In the larval zebrafish spinal cord, individual axon subtypes supported distinct nascent sheath growth rates and stabilization frequencies. Oligodendrocytes ensheathed individual axon subtypes at different rates during a two-day period after initial axon wrapping. When descending reticulospinal axons were ablated, local spinal axons supported a constant ensheathment rate despite the increased ratio of oligodendrocytes to target axons.

Conclusion: We conclude that properties of individual axon subtypes instruct oligodendrocyte behaviors during initial stages of myelination by differentially controlling nascent sheath growth and stabilization.

Keywords: Glia, Oligodendrocyte, Myelination, Myelin sheath

\section{Background}

Ensheathment of nerve axons with myelin is an essential process during vertebrate neural development. Oligodendrocytes are specialized central nervous system (CNS) ensheathing cells that extend multiple membrane processes to sample candidate axons, initiate and perform spiral axon wrapping, then extend along axons to form a mature myelin sheath (reviewed by $[1,2]$ ). Alternatively, oligodendrocytes can retract and prune myelin sheaths [3-5], as well as modify the thickness of individual sheaths in response to external stimuli [6]. These

* Correspondence: jhhines@winona.edu

Biology Department, Winona State University, Winona, MN, USA complex steps take place over the course of days or weeks, and ultimately are responsible for mature myelin sheaths being positioned at the right location, time, and parameters [7-9]. Numerous distinct cell behaviors and specialized mechanisms are deployed during these steps, including dynamic process extension and retraction, cell-type recognition, cell adhesion, and protrusive myelin membrane growth for concentric wrapping and extension along axons (reviewed by [10-12]). No single axonal factor has been identified that is required for myelination, but many regulate the abundance, length, and thickness of myelin sheaths $[9,12-15]$. How and when these factors regulate stage-specific 
oligodendrocyte cell behaviors, leading to the complex myelination patterns observed in the CNS, is not well understood.

The discoveries that cultured oligodendrocytes form immature myelin onto chemically fixed axons and synthetic fibrils indicate that oligodendrocytes possess an intrinsic ensheathment program that is capable of executing at least some of the cell behaviors and stages of myelination [16-19]. The extent to which oligodendrocytes utilize axon-derived cues in vivo is less clear, but it is possible that the first steps and behaviors mediating sheath formation and initial stabilization could occur without molecular instruction from axons. In vitro, oligodendrocytes require synthetic fibrils to possess a minimal diameter of $0.3 \mu \mathrm{m}$ in order to initiate sheath formation [17]. Regulation of axon diameter by radial growth (or lack thereof) could therefore define axons as either permissive or non-permissive for myelination, after which continued wrapping, stabilization, and sheath maturation could be supported by axon-derived molecular cues. In this way, an oligodendrocyte-intrinsic ensheathment program could mediate initial sheath formation based on the permissive diameter of an axon, followed by axon-derived signals that instruct later oligodendrocyte behaviors to complete and refine the myelination process [20]. In support of this model, an artificial increase in axon diameter by genetic deletion of PTEN is sufficient to induce oligodendrocytes to myelinate cerebellar granule cell axons, which are normally unmyelinated [21]. Furthermore, a recent study demonstrated that oligodendrocytes continue to initiate myelination in the optic nerve in the absence of dynamic neuronal signaling [22]. Numerous studies collectively support the notion that oligodendrocytes are intrinsically programmed to wrap any axon of suitable caliber, but the extent to which these mechanisms facilitate the unique spatial and temporal profiles of CNS myelination in vivo is less clear, and may be region- or axon subtype-specific. The purpose of this study was to evaluate whether initial sheath extension, sheath stabilization, and the rate at which individual axons become myelinated is modified by the axon subtype involved or is an intrinsic property of oligodendrocytes.

The CNS comprises many distinct neuronal subtypes, each possessing specific genetic profiles, structural morphologies, and functional connectivity. Oligodendrocytes stereotypically myelinate some axon subtypes but not others, but the cellular interactions forming this heterogenous myelin landscape are unknown. In this study we provide evidence that the earliest steps of sheath formation, stabilization, and growth are influenced by properties of individual axons. Using in vivo imaging in the larval zebrafish spinal cord, we found that distinct axon subtypes differentially controlled the rate of nascent sheath growth and initial stabilization. Consequently, the myelin ensheathment of distinct axon subtypes proceeded at different rates during the $48 \mathrm{~h}$ following initial wrapping. When a subset of target axons was ablated, remaining axon subtypes maintained consistent ensheathment rates despite the increased ratio of oligodendrocytes to myelin-competent axons. Taken together, these findings support the hypothesis that properties of individual axons are sufficient to influence or override the oligodendrocyte-intrinsic ensheathment program by regulating initial sheath growth and stabilization at the onset of myelination in vivo.

\section{Methods}

\section{Zebrafish lines and husbandry}

All animal work performed in this study was approved by the Institutional Animal Care and Use Committee at Winona State University. Zebrafish embryos were raised at $28.5^{\circ} \mathrm{C}$ in egg water $(0.0623 \mathrm{~g} / \mathrm{L}$ Coralife marine salt $)$ and staged according to hours post-fertilization or morphological criteria. The sex of animals was not determined. Transgenic lines used in this study included $\operatorname{Tg}(\operatorname{sox} 10: R F P$ CaaX $)^{v v 234}, \quad T g(\operatorname{sox} 10: T a g R F P-T)^{c o 26}, \quad T g(n k x 2.2 a: E G F P-$ CaaX) ${ }^{\text {vu16, }} \operatorname{Tg}\left(\right.$ phox2b:EGFP) w37, $\operatorname{Tg}\left(\right.$ phox2b:GAL4) ${ }^{c 021}$, $\mathrm{Tg}$ (tbx16:EGFP) иааб/812c, $\operatorname{Tg}$ (isl1[ss]:Gal4-VP16, UAS: DsRed $)^{z f 234}$, and $\operatorname{Tg}(\text { pitx2c:EGFP })^{z y s}$.

\section{Microscopy and quantitative image analysis}

Embryos or larvae were embedded in 1\% low-melt agarose using tricaine (MS-222) anesthesia $(3.48 \mathrm{mM}$, Pentair). All images were acquired or rotated to show lateral views with anterior left and dorsal up. Confocal microscopy was performed using an Olympus IX-81 equipped with a disk spinning unit (DSU), $60 \times 1.3 \mathrm{NA}$ silicone immersion objective (unless otherwise stated), LED illumination with narrow bandpass filters, and a Hamamatsu Orca-R2 CCD camera. Z-intervals were $400 \mathrm{~nm}$ and individual camera pixel sizes were $107.5 \mathrm{~nm}$, or if $2 \times 2$ binning was required for fluorescence detection, $215 \mathrm{~nm}$. All image analysis was performed using CellSens (Olympus) and NIH ImageJ software. Images were deconvolved using 3D iterative deconvolution (CellSens, Olympus) and all projection images were produced using maximum intensity.

\section{Determination of axon subtype-specific ensheathment, sheath growth rates, and pruning}

To determine overall ensheathment rates, a baseline time point was first defined for each individual axon subtype as a specific spinal cord segment where axon wrapping had initiated in the majority of larvae imaged. This baseline $(t=0)$ was determined by the presence of a low but consistent level of sheath initiation on transgene-labeled axons. Subsequent images were 
acquired 24 and $48 \mathrm{~h}$ later within the same spinal cord segment. For each image, the percent of individual axon length ensheathed was determined by dividing the sum of all sheath lengths (with GFP/RFP colocalization) by the total axon length within a field of view. For measurements of individual sheath growth and pruning, we collected images of the same nascent sheath at $t=0$ and $t=3 \mathrm{~h}$. A nascent sheath was defined as a structure with processes wrapped around the entire circumference of an axon in $x-y$ and orthogonal views, containing a clearly defined lumen devoid of oligodendrocyte-channel fluorescence, and myelinating internodes $2-5 \mu \mathrm{m}$ in length. During the 3-h period between image acquisitions, fish were incubated at $28.5^{\circ} \mathrm{C}$ while embedded in $1 \%$ lowmelt agarose under tricaine anesthesia. Sheath growth rate measurements were restricted to sheaths that were present at both time points. A pruning event was defined as a full retraction such that at $\mathrm{t}=3 \mathrm{~h}$ no interaction remained, or the remaining interaction lacked both parallel processes and a lumen.

\section{Determination of myelin sheath distribution on individual axon subtypes during early myelination}

We first measured the percent of all myelin associated with the Mauthner axon, dorsal to the Mauthner, and ventral to the Mauthner axon. To accomplish this, the combined sheath lengths within the three aforementioned dorsal-ventral domains were summed and then divided by the combined lengths of all sheaths within the spinal hemisegment. Images were acquired in somites 24-25 (restricted to larvae possessing 10-50 sheaths per domain) at $96 \mathrm{hpf}$ (hours post-fertilization). Individual sheaths within each of the three domains were next assigned to individual axon subtypes. First, axon subtypes were assigned a domain of residence based upon their anatomical position (e.g. $i s l 1^{+} \mathrm{RB}$ is dorsal/medial). Next, we determined the lengths of all sheaths within the resident domain. We separated these values into two categories (yes/no) based on whether the sheath exhibited RFP/GFP colocalization with an axon marked by the subtype-specific reporter line. The total length of sheaths with RFP/GFP colocalization was then divided by the combined length of all sheaths within the resident domain. Images were acquired at 98-105 hpf. Images of ventral axon subtypes were acquired at somite 27 , whereas images of dorsal/medial subtypes were acquired at somite 21 to account for the slight developmental delay of myelination within the dorsal spinal cord.

Ablation procedures, validation, and imaging experiments A sharp-edged ablation needle was generated from pulled capillary glass and adjusted to $60 \mu \mathrm{m}$ diameter using \#5 forceps. Zebrafish embryos staged at 2-3 days post-fertilization (dpf) under tricaine anesthesia were transferred atop Sylgard-coated petri dishes and aligned anterior left and dorsal up, with minimal egg water, under a dissecting microscope. The ablation needle was held roughly vertical and perpendicular to the embryo (60-75 degree angle) and inserted through the spinal cord at somite 7. Care was taken to avoid desiccation of the animal or injury to the notochord. Animals were immediately transferred to egg water and incubated at $28.5^{\circ} \mathrm{C}$. Behavior was assessed at $24 \mathrm{~h}$ post-injury (hpi) and immediately prior to data acquisition. Animals exhibiting any signs of spontaneous swim in a period of 20 min, response to light stimulation, or response to touch (pin tool) near the otolith were discarded. Unless otherwise indicated, the $T g$ (pitx2c:EGFP) reporter was crossed into the background of all animals used for experiments to visually verify the absence of distal reticulospinal axon segments in the region of interest prior to data acquisition. Because the absence of spontaneous swim behavior and complete morphological reticulospinal axon ablation corresponded (41 of 41 animals), behavioral criteria were used to select animals for imaging of isl1 [ss] $]^{+}$RohonBeard (RB) axons because $n k x 2.2 a: E G F P-C a a X^{+}$cells prevented observation of $\mathrm{GFP}^{+}$reticulospinal axons in the ventral spinal cord. Non-injured, stage-matched siblings were used as controls.

Oligodendrocyte-lineage cell counts and myelin sheath counts were performed at $96 \mathrm{hpf}$ (corresponding to 24 hpi), and images were acquired within somites $24-26$ and 24-25, respectively. For oligodendrocyte-lineage cell counts, images were acquired using a $20 \times 0.7 \mathrm{NA} \mathrm{ob-}$ jective. The domains were delineated by sheaths wrapping the $t b \times 16^{+}$CoPA axon, whose diagonal ascending portion traversed the medial domain then projected horizontally along the dorsal domain, and by sheaths wrapping the Mauthner axon, which separated the medial domain (sheaths above) from the ventral domain (sheaths below). Sheaths wrapping the Mauthner axon were excluded from analysis. Local spinal neuron cell body and axon counts were performed at $96 \mathrm{hpf}(24 \mathrm{hpi})$ and images were acquired at or posterior to somite 20 for isl1[ss] $]^{+}$neurons and within somites 16-17 for tbx $16^{+}$neurons.

For assessment of isl1[ss] $]^{+} \mathrm{RB}$ sensory axon ensheathment, larvae were imaged at $96 \mathrm{hpf}(24 \mathrm{hpi})$. All sheaths at or posterior to somite 21 were counted and imaged. Selection of somite 21 was based on the absence of sheath initiation at this segment at the time of injury (72 hpf), enabling later assessment of sheaths that had formed in an environment lacking descending reticulospinal axons. For assessment of $t b x 16^{+} \mathrm{CoPA}$ axon ensheathment, the distribution of $t b \times 16^{+} \mathrm{CoPA}$ neurons necessitated alterations in somite of data acquisition. Because the $T g(t b x 16: E G F P)$ reporter line marks relatively 
few CoPA axons in posterior spinal cord somites 24-26 (see Additional file 1: Fig. S1a), we shifted investigation anteriorly to somites 16-17, where CoPA axons were more abundant. The anterior shift in somite acquisition required a concomitant shift in time points to earlier developmental stages (injury was performed at $48 \mathrm{hpf}$ as opposed to $72 \mathrm{hpf}$ in isl1[ss $]^{+} \mathrm{RB}$ experiments). Larvae were imaged at $72 \mathrm{hpf}$ and $84 \mathrm{hpf}(24 \mathrm{hpi}$ and $36 \mathrm{hpi})$. To verify injury performed at the earlier $48 \mathrm{hpf}$ time point still resulted in the complete absence of reticulospinal axons within the posterior spinal cord at both 24 and $36 \mathrm{hpi}$, the $\operatorname{Tg}($ pitx $2 c: E G F P)$ reporter line was crossed into all animals for internal validation. Zebrafish embryos have not initiated myelination by $48 \mathrm{hpf}$, therefore any myelin that we observed had formed in the absence of reticulospinal axons.

\section{Determination of axon diameters using super resolution radial fluctuations (SRRF) imaging}

For acquisition, an individual axon was located and an image series comprising 100 single z-plane images with minimal time delay (rate of $\sim 12$ frames per second) was obtained by standard widefield fluorescence microscopy. A corresponding single z-plane GFP/RFP image depicting the location of myelin on the axon was also obtained for reference. The 100 frame series was imported into Fiji and processed using the NanoJ-SRRF plugin with TRA and TRPPM temporal settings [23]. Fourier ring calculation (FRC), performed using the Fiji BIOP plugin, reported a functional resolution of $129.5 \mathrm{~nm}$ in our hands that is comparable to a previous report of $123 \mathrm{~nm}$ in the larval zebrafish spinal cord [24]. Auto local thresholding was then performed using the Bernsen method. Myelinated and non-myelinated regions of interest were identified, and axon diameter was determined by dividing the ROI area (based on all pixels exceeding threshold) by the ROI length.

\section{Quantification and statistical analysis}

In instances where the data were normally distributed, we used an unpaired, two-tailed t-test for hypotheses concerning differences between two means. In instances where data were not normally distributed, we preferentially utilized non-parametric tests to compare distributions: the Mann-Whitney test when comparing distributions of two groups or the Kruskal-Wallis test for comparing distributions of more than two groups. When the Kruskal-Wallis test was significant, Dunn's method was used to adjust for multiple comparisons to determine where differences existed. A chi-square test was used to determine if individual axon subtypes supported sheath pruning versus stabilization (binary outcomes) by comparing percentages of outcomes across groups. Tukey-adjusted pairwise comparisons were then used to determine where differences existed. In order to determine if myelination rate differed across the axon subtypes, an analysis of covariance (ANCOVA) was fit, allowing for an interaction between time and axon subtype. Tukey-adjusted pairwise comparisons were used to assess potential myelination rate differences between individual subtypes and generate $p$-values. Fisher's exact test was used to determine whether the presence of barriers differed across axon subtypes, with Bonferroni correction to adjust for multiple pairwise comparisons. Simple linear regression was used to determine whether sheath growth rate correlated with change in axon diameter. All statistical analyses were performed using Prism 6 (GraphPad), JMP Pro 14.0, or R Studio 1.1.453. In all figures, error bars represent mean \pm standard error of the mean (SEM).

\section{Results}

In larval zebrafish spinal cord, each hemisegment possesses multiple differentiated oligodendrocytes that collaborate to form stereotyped myelination patterns. Single oligodendrocytes can extend processes into and form myelin sheaths within both dorsal and ventral white matter tracts, which are composed of unique axon populations. Each individual oligodendrocyte therefore myelinates multiple target axon subtypes. To determine how oligodendrocyte behaviors are influenced by different axon subtypes, we first identified myelin-competent target axons within the larval zebrafish spinal cord. Previous studies established reticulospinal neurons and commissural primary ascending (CoPA) interneurons as myelinated axon subtypes $[5,25]$. In the spinal cords of 3-5 dpf larvae, we observed $\mathrm{Tg}(\mathrm{tbx}$ 16:EGFP) reporter expression restricted to CoPA neurons [26]. CoPA axons projected ipsilaterally toward the ventral floor plate, crossed the ventral midline, and extended contralaterally and anteriorly into and within the dorsal longitudinal fasciculus (DLF) (Additional file 1: Fig. S1a). When outcrossed to $\mathrm{Tg}(\operatorname{sox} 10: R F P-C a a X)$, which marks oligodendrocyte processes with membrane-tethered RFP, we observed ensheathment of $\mathrm{EGFP}^{+} \mathrm{CoPA}$ distal axon segments located within the DLF (Fig. 1a). Oligodendrocytes did not myelinate ipsilateral axon segments, but myelinated all contralateral axon segments. We also utilized the $\operatorname{Tg}$ (isl1[ss]:Gal4, UAS:DsRed) reporter line to label Rohon-Beard (RB) neurons, a second population of local spinal neuron axons. RB sensory neuron axons also extended within the DLF, adjacent but immediately dorsal to the position of CoPA axons. When outcrossed to $\operatorname{Tg}(n k x 2.2 a: E G F P-C a a X)$, which marks oligodendrocyte processes with membrane-tethered EGFP, we observed ensheathment of DsRed ${ }^{+}$RB axons (Fig. 1a) [25, 27].

Spinal cord oligodendrocytes myelinate axons projecting from both local spinal cord neurons and descending 
a
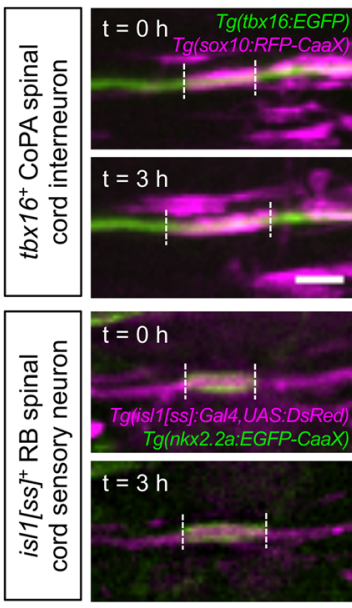

C
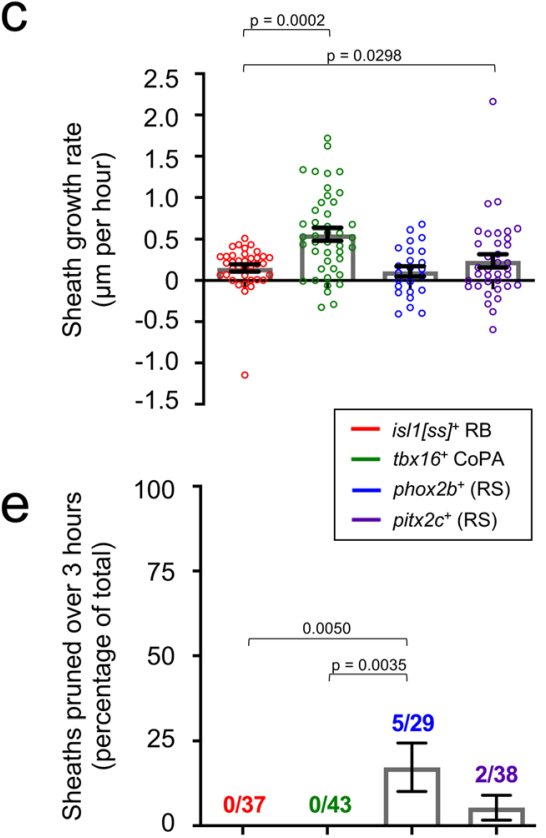

$\mathrm{b}$
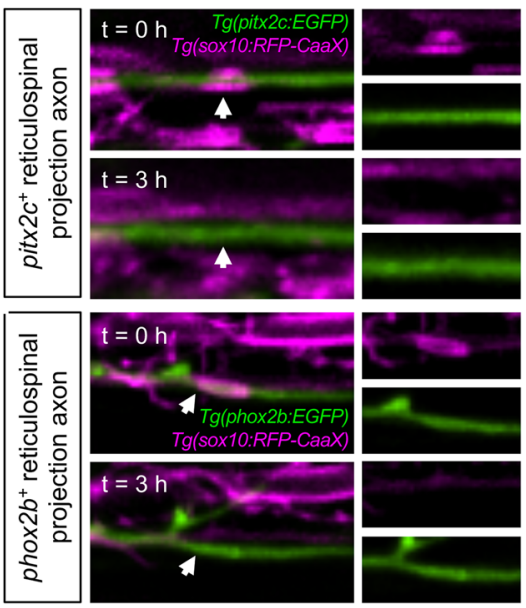

d
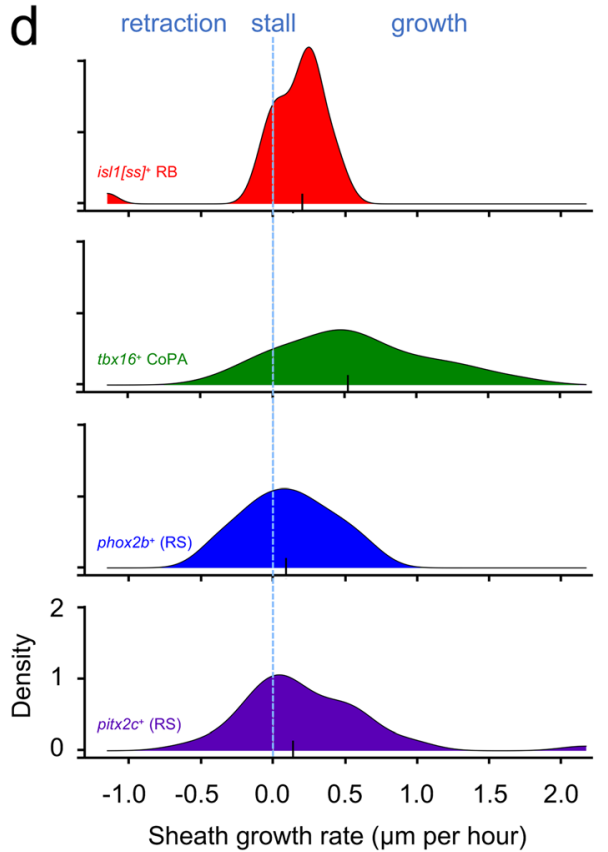

Fig. 1 Individual sheath extension rates and pruning frequencies are axon subtype-specific. a-b Lateral view images of the larval spinal cord show sheath extension (a) and sheath pruning (b) during a 3-h time course. Transgenic reporter expression (indicated) marks individual axons or oligodendrocyte membranes. Dashed lines illustrate individual sheath lengths and arrowheads denote sheath pruning events. Images are confocal maximum z-projections with dorsal up and anterior left. Scale bar $=3 \mu \mathrm{m}$. c-d Plots show mean growth rates (c) and distributions of individual sheath growth rates $(\mathbf{d})$. For $(\mathbf{c})$, error bars represent the mean \pm SEM. For $(\mathbf{d})$, the $y$-axis approximates the density of data points that exhibited the corresponding growth rate. Larger $y$-values represent a larger proportion of data points exhibiting this growth rate. Black ticks indicate medians. $\mathrm{n}$ (\# of sheaths) $=24\left(\right.$ phox $\left.2 b^{+}\right), 43\left(t b \times 16^{+}\right), 36\left(\right.$ pit $\left.\times 2 c^{+}\right), 37\left(\right.$ is $\left.17[s s]^{+}\right)$. e Quantification of the percentage of total sheaths that pruned during a 3-h time course grouped by axon subtype. $n$ (sheaths) $=29\left(\right.$ phox $\left.2 b^{+}\right), 43\left(\right.$ tbx $\left.16^{+}\right), 38\left(\right.$ pitx $\left.2 c^{+}\right), 37\left(\right.$ is $\left./ 7[s s]^{+}\right)$. Overall chi-square test of difference in percent pruning $=0.0025$. Pairwise $p$-values were generated using Dunn's multiple comparisons (c) or using Tukey-adjusted pairwise comparisons (e)

reticulospinal neuron populations. We previously identified phox $2 b^{+}$neurons as hindbrain reticulospinal axons that become myelinated within and along the length of the ventral spinal cord medial longitudinal fasciculus (Fig. 1b, Additional file 1: Fig. S1c) [5]. To identify additional reticulospinal myelinated axon subtypes, we crossed $T g(\operatorname{sox} 10: R F P-C a a X)$ to the $\operatorname{Tg}($ pitx2c:EGFP) reporter line, which marks the rostral-most reticulospinal neurons of the nucleus of the medial longitudinal fasciculus (nMLF) [28]. nMLF cell bodies, resident to the ventral midbrain, projected axons into and posteriorly across the length of the ventral spinal cord (Additional file 1: Fig. S1d), consistent with an established role for the nMLF in coordinating locomotor responses to visual input [2931]. In 3-5 dpf larvae, we observed $\operatorname{sox} 10^{+}$sheaths 
wrapping $\mathrm{EGFP}^{+}$nMLF axons located in the ventral spinal cord (Fig. 1b).

\section{Subtype-specific rates of initial ensheathment and sheath pruning}

If the early events of myelination are mediated by an oligodendrocyte-intrinsic ensheathment program, we predicted that the behavior of newly formed (nascent) individual myelin sheaths would be independent of the axon subtype ensheathed. In contrast, if unique properties of individual axons play an instructive role during these early stages, we hypothesized that initial sheath growth and stabilization would differ depending on the axon subtype being wrapped. To distinguish between these possibilities, we identified nascent sheaths wrapping transgene-labeled axons and tracked progression over three hours (Fig. 1). We defined nascent sheaths as those less than $5 \mu \mathrm{m}$ in length. This criterion was based on the previous finding that sheaths that are initially stabilized extend to greater than $5 \mu \mathrm{m}$ within three hours or less [5]. Therefore, in our study, observed sheaths less than $5 \mu \mathrm{m}$ in length had most likely formed within the previous three hours. Nascent sheaths wrapping $t b x 16^{+}$ CoPA axons extended at an average rate of $0.6 \mu \mathrm{m} / \mathrm{h}$ (Fig. 1a, c). In comparison, other sheaths within the DLF that wrapped isl1[ss] $]^{+} \mathrm{RB}$ sensory axons extended at a comparatively slower average rate of $0.2 \mu \mathrm{m} / \mathrm{h}$ (Fig. 1a, c). We observed similar average growth rates among nascent sheaths wrapping pitx $2 c^{+}$and phox $2 b^{+}$ventralresiding reticulospinal axons $\left(0.1 \mu \mathrm{m} / \mathrm{h}\right.$ for phox $2 b^{+}$and $0.2 \mu \mathrm{m} / \mathrm{h}$ for $p i t x 2 c^{+}$, Fig. 1c). In addition to subtypespecific differences in the average sheath growth rate, we also noted subtype-specific differences in the distribution of the individual sheath growth rates that composed the average (Fig. 1d). Within the dorsal tract, isl1[ss] ${ }^{+} \mathrm{RB}$ sensory axons predominantly exhibited slow sheath growth (mostly $0.2 \mu \mathrm{m} / \mathrm{h}$ or less), whereas $t b x 16^{+}$CoPA axons had the ability to support more rapid sheath growth (up to $1.7 \mu \mathrm{m} / \mathrm{h}$ ). Within the ventral tract, sheaths wrapping both pitx $2 c^{+}$and phox $2 b^{+}$reticulospinal axons exhibited a tendency to stall or retract, however the population of sheaths that did extend largely did so at a moderate rate (combined median $=0.3 \mu \mathrm{m} / \mathrm{h}$ ). Therefore, although both dorsal sensory and ventral reticulospinal axons had comparable average sheath growth rates $(0.1-0.2 \mu \mathrm{m} / \mathrm{h})$, sheaths wrapping dorsal $\mathrm{RB}$ sensory axons achieved this through slow growth, whereas sheaths wrapping ventral reticulospinal axons performed a combination of retractions, stalls, and moderate growth to achieve the same average growth rate.

We next addressed whether the subtype-specific differences in nascent sheath extension rates could be explained by the length of axon available for myelination. Available axon space is restricted by neighboring sheaths and potentially restricted by other axonal morphological features such as collateral branches (filopodia) and varicosities. To evaluate this, we marked sheaths, filopodia, and varicosities (collectively referred to as "barriers") within $6.5 \mu \mathrm{m}$ segments directly adjacent to each side of the nascent sheath $(6.5 \mu \mathrm{m}=$ maximum nascent sheath extension during 3-h assay). We then examined the relationship between nascent sheath growth rate and the presence of barriers within the window, across subtypes, using Fisher's exact test and Bonferroni correction for multiple comparisons. These comparisons revealed that $35 \%$ of nascent sheaths wrapping CoPA axons had a barrier within the $6.5 \mu \mathrm{m}$ window, which was a larger proportion than the $3 \%$ of nascent sheaths wrapping $R B$ axons $(P=0.003)$. In contrast, the proportion of sheaths wrapping CoPA axons possessing nearby barriers (35\%) was less than (but without statistical significance) that observed for phox $2 b^{+}$axons (59\%; $\left.P=0.159\right)$, and significantly lower than that observed for pitx $2 c^{+}$axons (65\%; $P=0.036)$. Can these differences explain subtypespecific individual sheath growth rates? The growth rate differences between nascent sheaths wrapping $\mathrm{RB}$ and CoPA axons cannot be explained by more available axon space on CoPA axons because if this were the case, we would have expected a higher percent of barriers on $\mathrm{RB}$ axons. Instead, fewer sheaths wrapping $\mathrm{RB}$ axons had nearby barriers, yet these sheaths extended at a slower average rate compared to sheaths wrapping CoPA axons. While the prevalence of nearby barriers on phox $2 b^{+}$ axons was higher than on CoPA axons, this did not reach significance, again reducing the likelihood of this mode of regulation. The prevalence of barriers adjacent to sheaths wrapping pitx $2 c^{+}$axons was higher than that of CoPA axons, which could explain the comparatively lower growth rates for sheaths wrapping pitx $2 c^{+}$axons. However, we noted that all but one of the sheaths wrapping pitx $2 c^{+}$axons extended a short distance of less than $3.5 \mu \mathrm{m}$. Because the majority of barriers observed were further removed than $3.5 \mu \mathrm{m}$, we conclude that these barriers were unlikely to have substantially regulated the extension rate of sheaths wrapping pitx $2 c^{+}$axons. Taken together, these results do not suggest that available axon space is a major driver of subtype-specific sheath behavior.

Previous studies indicate that some initial axon wrapping attempts can undergo retraction and pruning, motivating us to ask whether the outcome of sheath stabilization versus pruning could be axon subtypedependent [3-5]. During the 3-h imaging window, oligodendrocytes stabilized $100 \%$ of sheaths wrapping $t b \times 16^{+}$ CoPA axons (43/43) and isl1[ss] ${ }^{+}$RB sensory axons (41/ 41) (Fig. 1a, e). In contrast, $10.4 \%$ reticulospinalpopulation axons were pruned during the imaging period (5/29 wrapping phox $2 b^{+}, 2 / 38$ wrapping pitx $\left.2 c^{+}\right)$ 
(Fig. 1b, e). We also addressed the possibility that either pruning frequencies or nascent sheath growth rates correlated with changes in axon diameter during the 3-h imaging period and found no relationship $(P=0.731$ for sheath pruning, $P=0.361$ for sheath growth).

Do differential nascent sheath growth rates and pruning frequencies result in axon subtype-specific differences in overall ensheathment rate? We next tracked the initial 48-h progression of axon ensheathment on transgene-labeled subtypes. For each, we identified a baseline time point $(t=0)$ based on the earliest appearance of nascent sheaths within a defined spinal cord segment, and subsequently acquired images within the same spinal segment 24 and $48 \mathrm{~h}$ later. Oligodendrocytes did not increase the overall percent ensheathment of isl1[ss] ${ }^{+} \mathrm{RB}$ sensory axons over the 48 -h period post initial wrapping (Fig. 2a, c). In stark contrast, oligodendrocytes rapidly myelinated $t b x 16^{+} \mathrm{CoPA}$ axons and arrived at near complete ensheathment $(92.0 \pm 1 \%$ of total axon length) during the same 48 -h period post initial wrapping (Fig. 2b, c). In comparison to the near full ensheathment of $t b \times 16^{+}$CoPA axons observed $48 \mathrm{~h}$ post initial wrapping, oligodendrocytes ensheathed $44 \pm 5 \%$ and $33 \pm 9 \%$ of the length of pitx $2 c^{+}$and phox $2 b^{+}$spinal projection axons, respectively (Fig. 2c).

The initiation of myelination requires a threshold axon diameter to be met [17]. This led us to question whether differences in axon diameters could instruct the rate of axon ensheathment and account for the observed subtype-specific differences in myelination. To test this, we performed super-resolution radial fluctuations (SRRF) imaging to assess the diameters of individual axons across the same 48-h observation period used to evaluate rates of axon ensheathment in Fig. 2a-c. Axon diameter is variable along the length of individual axons, and we therefore reasoned that at any given developmental timepoint, the proportion of axon length exceeding the threshold diameter could vary by axon subtype. We therefore tested the prediction that subtype-specific differences in the rate of myelination can be explained by differences in the timing and onset of achieving threshold diameters. Specifically, we hypothesized that RB sensory and reticulospinal subtypes would possess fewer supra-threshold axon segments $(>0.3 \mu \mathrm{m})$ in comparison to more rapidly myelinated CoPA axons. However, we found that regardless of axon subtype, all individual axon segments that we measured exceeded a diameter of $0.5 \mu \mathrm{m}$. Because all axon segments exceed the threshold diameter, we conclude that the proportion of individual axon lengths exceeding threshold, and the timing of this process, cannot explain the observed differences in myelination rate between subtypes.

Although the requirement for a threshold diameter is well established, whether differences in diameter above this threshold can act instructively is less clear. If instructive, we predicted that oligodendrocytes would myelinate different axon subtypes at rates corresponding to their diameters. To address whether subtype-specific differences in axon diameter existed, we compared diameters using SRRF imaging at the onset of the 48-h observation period. CoPA axons exhibited the largest average diameter $(1.05 \mu \mathrm{m})$. In comparison, other subtypes examined were of slightly lower caliber (pitx $2 c=0.75 \mu \mathrm{m}$, phox $2 b=0.71 \mu \mathrm{m}$, and isl1 $\left.[s s]^{+} 0.61 \mu \mathrm{m}\right)$. These observations are compatible with the possibility that comparatively large diameters of CoPA axons could cause their more rapid myelination. However, using the same logic, these findings cannot explain why reticulospinal axons become myelinated more rapidly than RB sensory axons, because SRFF imaging did not detect a significant diameter difference between these subtypes (Fig.2c). We also evaluated the possibility that oligodendrocytes could survey and initiate myelination based on subtype-specific changes (increases) to local axon diameter at unmyelinated segments. If true, we predicted that CoPA axons would show the greatest increase in axon diameters from the onset to completion of the 48-h observation period, but in comparison, reticulospinal and RB sensory axon diameters would remain more constant. However, our SRRF axon diameter measurements detected no significant changes to diameters, for any axon subtype examined, across the 48-h observation period. Together, our observations fail to support the hypothesis that axon caliber or radial axon growth explain subtype-specific differences in myelination rate. Instead, our results indicate that properties of individual axon subtypes other than axon diameter control the initial rate of overall ensheathment by differentially regulating both individual sheath growth and stabilization.

\section{Alteration of target axon availability reveals subtype- specific control of initial ensheathment}

Previous studies indicate that zebrafish spinal cord oligodendrocytes survive and continue to myelinate in the absence of reticulospinal axons [32]. We recognized this as an opportunity to ask whether the rate that oligodendrocytes myelinate local spinal neuron axons can be influenced by the availability of reticulospinal axons, or is a hard-wired property imposed by the spinal neurons themselves. We first determined the proportion of myelin dedicated toward each axon population. By comparing the amount of myelin associated with individual transgene-labeled axons to the total myelin, we found that oligodendrocytes dedicate $61 \%$ of their myelinating resources to reticulospinal axons, but only $9 \%$ to $t b \times 16^{+}$ and $i s l 1^{+}$local spinal neurons (Fig. 3a). If reticulospinal target axons were removed, we predicted that oligodendrocytes would shift myelinating resources to remaining 


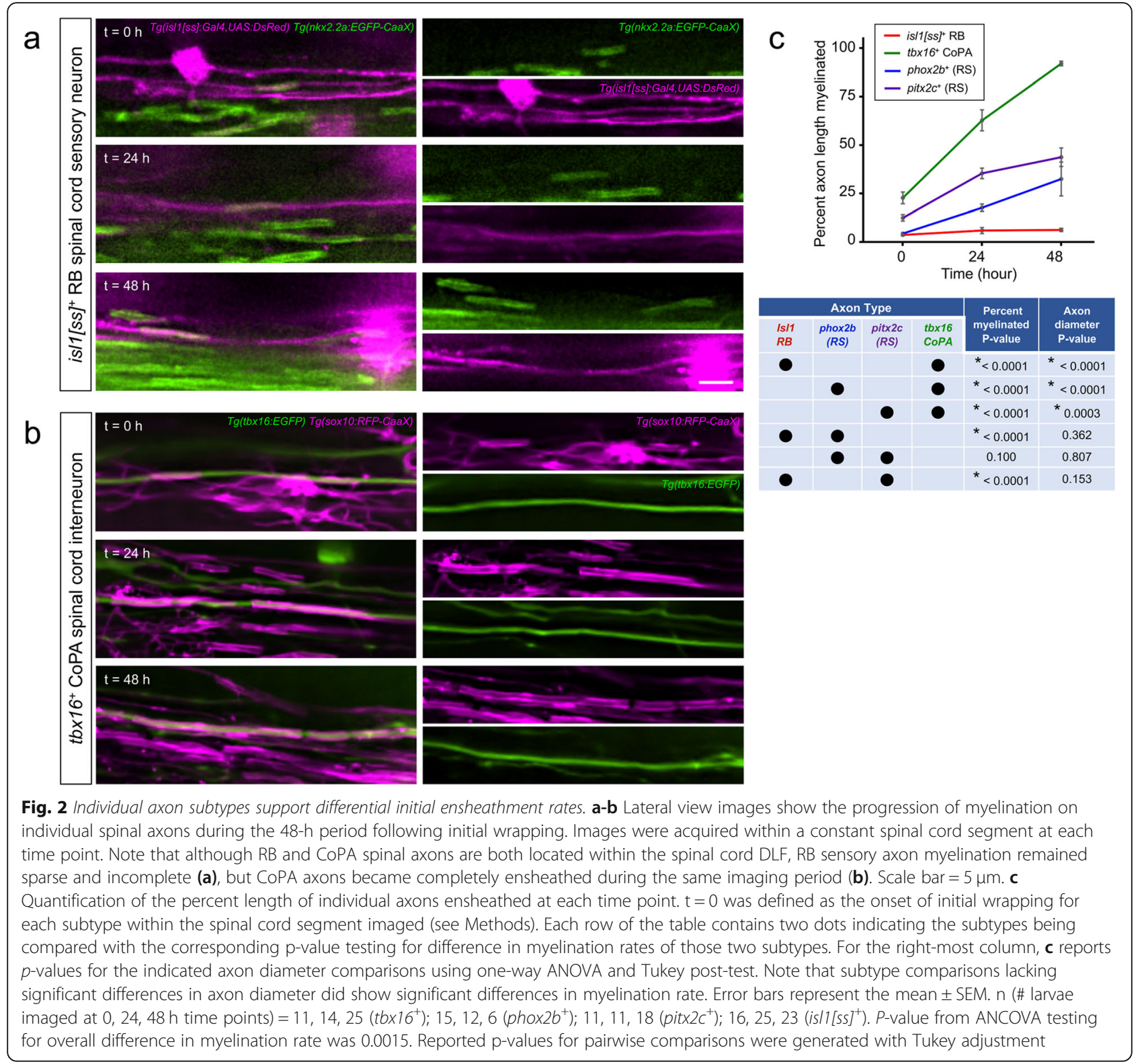

target axons if initial axon ensheathment is controlled by an oligodendrocyte-intrinsic program. In contrast, if initial axon ensheathment is governed by axon subtypespecific factors, we hypothesized that reducing the abundance of target axons would not alter the initial ensheathment of remaining target axons. In order to alter target axon availability, we took advantage of the different anatomical positions of reticulospinal versus local spinal sensory and interneuron populations. Specifically, because reticulospinal neuronal cell bodies reside in the midbrain and hindbrain, we reasoned that anterior axon ablation near the hindbrain-spinal cord boundary (somites 6-7) would remove distal reticulospinal axon segments in the posterior spinal cord (somites 16 or beyond) (Fig. 3b). We therefore used an anterior spinal cord injury approach to ablate reticulospinal axons in 72 hpf embryos (Additional file 1: Fig. S2). To verify that injured axons did not regenerate into posterior segments within $24 \mathrm{~h}$ we directly observed pitx $2 c^{+}$reticulospinal axons at $24 \mathrm{hpi}$. In all instances, we detected either complete axonal loss or remnants of axons consistent with Wallerian degeneration in posterior segments (Fig. 3c, Additional file 1: Fig. S2a). In comparison, in control larvae (non-injured siblings), we routinely detected 2-7 pit $x 2 c^{+}$nMLF axons per spinal hemisegment in posterior segments. In contrast to the complete loss of reticulospinal axons, we observed no changes to the number nor morphology of isl1[ss] $]^{+} \mathrm{RB}$ sensory or $t b x 16^{+} \mathrm{CoPA}$ neurons in posterior segments of injured larvae at $24 \mathrm{hpi}$ (Fig. 3c, Additional file 1: Fig. S2b). Reticulospinal 


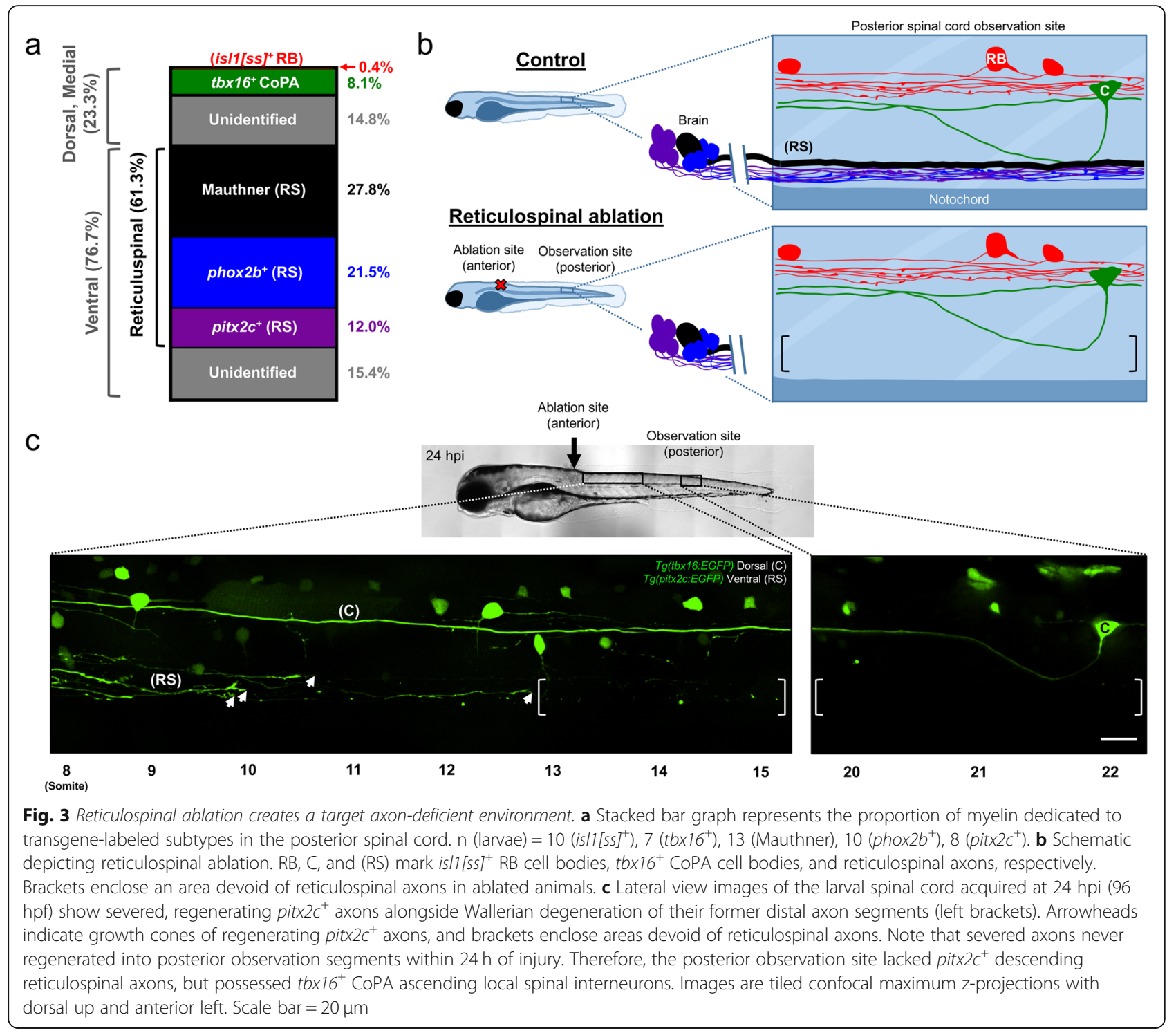

ablation caused a pronounced effect on animal behavior, because 41/41 animals completely lacking pitx $2 c^{+}$nMLF axons in posterior segments also lacked spontaneous swim behavior.

We next evaluated the validity of this approach to study myelin ensheathment within the posterior spinal cord. Within posterior segments, reticulospinal ablation caused a subtle reduction in sox10:TagRFP ${ }^{+}$ oligodendrocyte-lineage cells (somites 24-26, control group $=29 \pm 6$, ablation group $=23 \pm 5$ cells per hemisegment, see Additional file 1: Fig. S3a,b) and an overall reduction in number of $T g(\operatorname{sox} 10: R F P$-CaaX) myelin sheaths (somites 24-25, control group $=34 \pm 3$, ablation group $=20 \pm 3$ sheaths per hemisegment, Additional file 1: Fig. S3d). Notably, we observed a robust loss of sheaths in the ventral spinal cord, where reticulospinal axons normally reside, but no change to sheath numbers in the medial- or dorsalmost spinal cord regions (Additional file 1: Fig. S3d). Previous reports using a genetic rather than physical ablation to remove reticulospinal axons from the posterior spinal cord paralleled our observed decrease in oligodendrocyte-lineage cells and myelin sheath numbers [27, 32]. We observed reduced average sheath lengths across all spinal cord regions in ablated versus control groups, which could be the result of oligodendrocytes forming more but shorter sheaths on select remaining target axons [27] (Additional file 1: Fig. S3e). Taken together, we conclude that ablation creates an environment with a disproportionate reduction in oligodendrocyte-lineage cells (20.7\% reduction) compared to target axons (reticulospinal target axons receive $61.3 \%$ of myelin). Consequently, axon ablation increased the oligodendrocyte to target axon ratio, 
and thereby allowed us to test for axon subtype-level alterations in myelination in an environment with excess myelinating potential.

When the oligodendrocyte to target axon ratio is increased, does an oligodendrocyte-intrinsic program continue to maximize surplus myelinating resources by increasing the extent or rate that remaining local spinal neurons become myelinated? We next tested this possibility by interrogating ablation-induced changes to initial ensheathment at the subtypespecific level. Our earlier observations indicated that isl1[ss] ${ }^{+} \mathrm{RB}$ sensory axons are myelin-competent but are incompletely myelinated during larval stages (see Fig. 2a). Because overall ensheathment of isl1[ss] ${ }^{+} \mathrm{RB}$ axons remained stagnant from 3 to $5 \mathrm{dpf}$ under baseline conditions (Fig. 2a, c), we reasoned that reticulospinal ablation could shift and accelerate myelination onto RB axons. To test this, we crossed Tg(isl1[ss]:Gal4, UAS:DsRed) to the oligodendrocyte reporter $\operatorname{Tg}(n k x 2.2 a$ :EGFP-CaaX). At $72 \mathrm{hpf}$, oligodendrocytes within and posterior to somite 21 had not initiated myelination in the DLF, where RB axons resided (31/31 animals). We therefore performed anterior spinal cord injuries at $72 \mathrm{hpf}$ and observed RB axon ensheathment in all somites within and posterior to somite 21 at $96 \mathrm{hpf}$. Because ensheathment had not commenced at the time of ablation, all sheaths observed and counted within and posterior to somite 21 had formed after ablation of reticulospinal axons. During the 24-h period from 72 to $96 \mathrm{hpf}$, we observed an average of $2.8 \pm 0.5$ new sheaths formed onto RB axons in control animals (Fig. 4a, b). Notably, we detected no significant difference in the number nor lengths of new sheaths formed onto RB axons following ablation (Fig. 4b, c).

To further test the hypothesis that target axon removal would increase the rate of ensheathment of remaining local spinal neuron axons, we next investigated the ensheathment rate of individual $t b x 16^{+} \mathrm{CoPA}$ axons in the presence and absence of descending reticulospinal axons. In contrast to isl1[ss] $]^{+} \mathrm{RB}$ axons, CoPA axons become rapidly ensheathed during the 48-h period after initial wrapping (Fig. 2b, c), motivating us to assess multiple time points to identify potentially subtle changes to the rate of CoPA axon ensheathment. At both 24 and 36 hpi, CoPA axon myelination was indistinguishable between control and ablated groups (Fig. 5a-d). We observed no effect on the overall percentage length of individual CoPA axons myelinated nor the density of sheaths per individual CoPA axon, together indicating that oligodendrocytes did not respond by dedicating surplus myelin resources toward CoPA axons. Collectively, these findings indicate that when sub-populations of axons are removed, both $t b x 16^{+} \mathrm{CoPA}$ and isl1[ss] $]^{+} \mathrm{RB}$
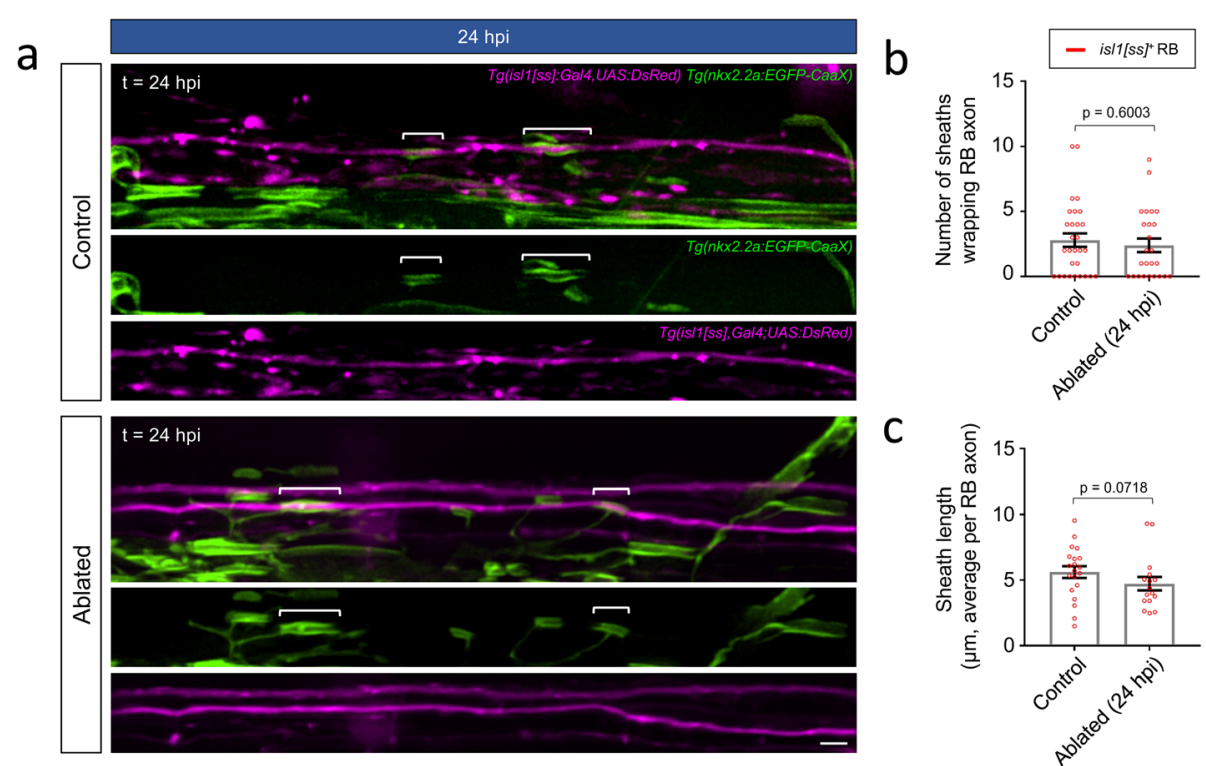

Fig. 4 RB spinal sensory neuron axons maintain minimal ensheathment in a target axon-deficient environment. a Lateral view images of the posterior spinal cord show ensheathment of RB sensory axons in control (non-injured siblings) and ablated larvae (24 hpi, 96 hpf). Note that oligodendrocytes had not yet formed sheaths within this spinal cord segment at the time of ablation, and therefore all sheaths observed in the ablated group were formed in a reticulospinal axon-devoid environment. Brackets indicate individual sheaths wrapping RB axons. Scale bar = $5 \mu \mathrm{m}$. b Quantification of RB axon ensheathment. Scatter plot points represent the total number of sheaths wrapping is/1[ss]:DsRed ${ }^{+}$RB axons in the posterior spinal cord of individual larvae (posterior to somite 2). n (larvae) = 29 (control), 25 (ablated). c Quantification of average sheath lengths. Scatter plot points represent the average length of all sheaths wrapping RB axons within an individual larva. $\mathrm{n}$ (larvae, total sheath \#) = 20, 80 (control) and 16, 60 (ablated). For $\mathbf{b}$-c, error bars represent mean \pm SEM and $p$-values report Mann-Whitney test 


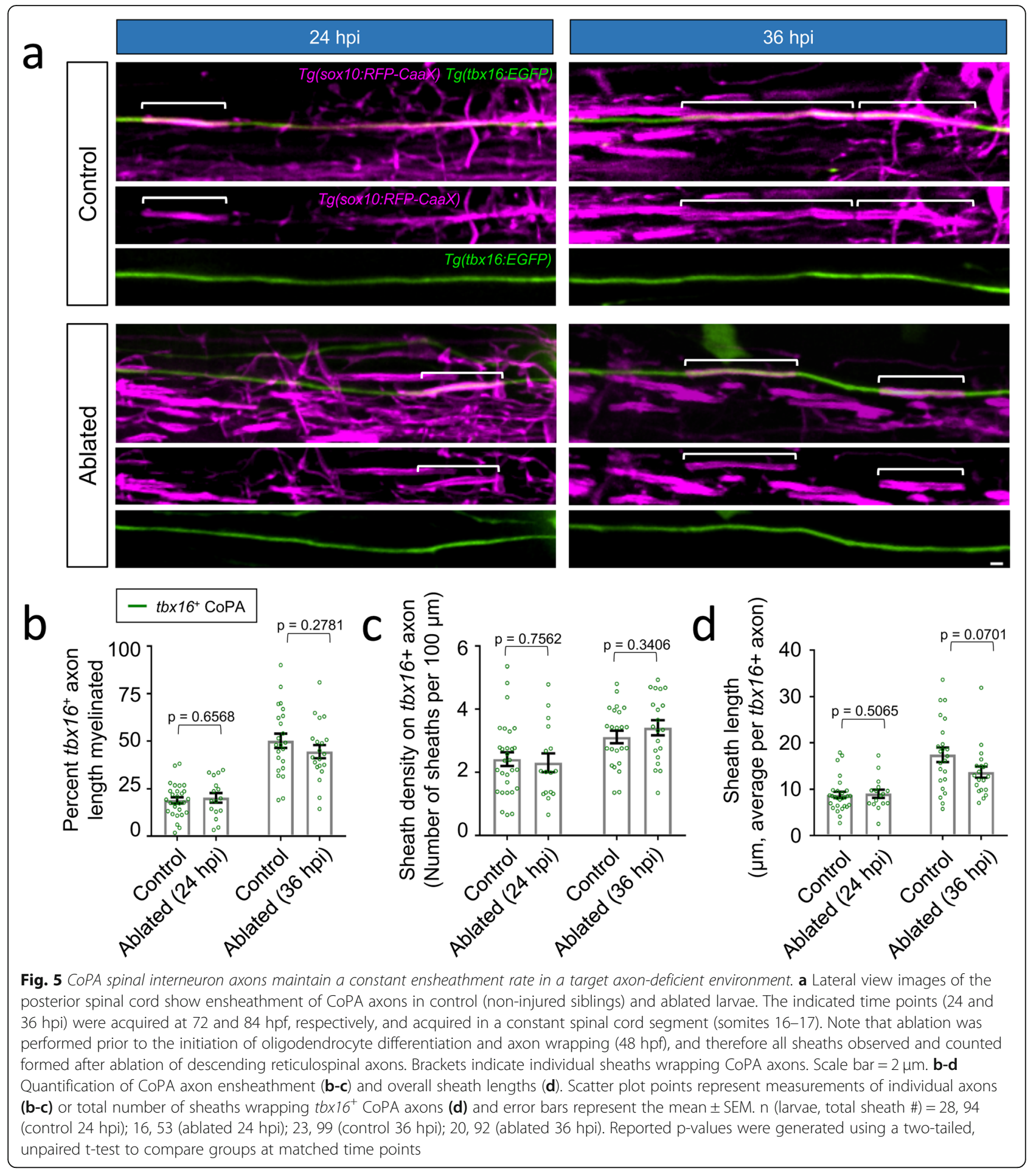

axons impose a defined rate of ensheathment, and oligodendrocytes do not adaptively extend longer sheaths onto these subtypes.

\section{Discussion}

Oligodendrocytes possess the ability to wrap and form immature myelin without active instruction from axons or synthetic substrates $[16-19,33]$. This raises the possibility that during normal neural development, oligodendrocytes deploy an intrinsic ensheathment program followed by axon-dependent and adaptive refinement of myelination [20]. In this sequential model, oligodendrocyte processes would indiscriminately wrap segments of any target axon competent for myelination. During this 
first intrinsic stage, any regional differences in the properties of myelin sheaths, such as sheath lengths, could be explained by the developmental origin and heterogeneity of oligodendrocytes and physical cues such as axon caliber [34]. At a later point during development or adulthood, oligodendrocytes would initiate an adaptive phase whereby axon-derived signals over-ride the oligodendrocyte-intrinsic ensheathment program, and further modify myelin sheath parameters such as length and thickness. Our findings add to a growing body of studies, discussed at length below, which collectively modify or fail to support the sequential model of oligodendrocyte-intrinsic myelination followed by adaptive refinement. If there are two sequential phases, our data indicate that the first phase is not solely mediated by an oligodendrocyte-intrinsic program, but rather is influenced by properties of individual axons. We found that during the initial hours of nascent sheath existence, properties of individual axons differentially influence sheath growth and stabilization, and consequently the rate that individual axon subtypes become myelinated. In this way, the oligodendrocyte-intrinsic ensheathment program and axonal regulators cooperate to determine which axons become myelinated and to what extent. We also show that properties of individual axons are sufficient to prevent changes to their rate of myelination, suggesting that axons autonomously control their own rate of myelination. Taken together, our findings support a cooperative and concurrent model, where from the onset of axon initial wrapping and sheath biogenesis, axonderived signals override the oligodendrocyte-intrinsic program to direct or restrict the early growth and stabilization of nascent sheaths.

\section{Axonal influence on oligodendrocyte behavior during initial wrapping and myelination}

If myelination is a multi-step process mediated by separable control mechanisms and cell behaviors, when and how do axonal signals participate in CNS myelination? Many neuronal factors influence oligodendrocyte development and myelination, but to date, no single neuronal factor has been identified as necessary to initiate and form myelin sheaths. Although it is possible that such factors will be identified by future studies, an alternative possibility is that oligodendrocytes will initiate wrapping and myelination of any candidate target axon that possesses a supra-threshold axon diameter $(>0.3 \mu \mathrm{m})$ and lacks inhibitory signals $[17,22]$, reviewed by $[9,13,14]$. For example, JAM2 was recently discovered as a somatodendritic molecule inhibitory towards myelination [35]. If JAM2 is sorted into the axons of some but not all neuronal subtypes, it would be positioned to regulate axon subtype-specific myelination patterns. Our studies do not directly address or exclude this possibility, and it will be important for future studies to determine if the transition from oligodendrocyte-axon contact to initial wrapping is influenced by axonal signals, or is solely mediated by an oligodendrocyte-intrinsic program in vivo. Regardless of whether axon identity influences the initiation of wrapping or subsequent sheath behavior, it is now clear that in both mouse and zebrafish models, individual axon subtypes have acquired distinct myelination patterns very early after the onset of myelination [36].

If the initial growth and stabilization of nascent sheaths is controlled by an oligodendrocyte-intrinsic program, we predicted that nascent sheath behavior would be independent of the axon subtype being myelinated. Instead, we observed that both nascent sheath growth and stabilization depended on the axon subtype being myelinated. For instance, spinal cord commissural axons $\left(t b \times 16^{+} \mathrm{CoPA}\right)$ supported more rapid sheath growth rates in comparison to sensory (isl1[ss] $]^{+}$) and reticulospinal axons (pitx $2 c^{+}$and phox $2 b^{+}$). Commissural and sensory axons consistently instructed nascent sheath stabilization, but in comparison, sheaths wrapping reticulospinal axons pruned more frequently. We interpret these observations to mean that axon subtype-specific factors over-ride the oligodendrocyte ensheathment program during the earliest steps of myelination. Because we directly observed the behaviors of nascent sheaths within their initial few hours after formation, if a purely oligodendrocyte-intrinsic program does temporally precede the emergence of axonal influences, our data indicate that these control mechanisms would be separated by only a few hours or less. One alternative explanation could be that the axon subtypes observed are surrounded by distinct extracellular matrix molecules, which could thereby influence the behavior of oligodendrocyte processes and nascent sheaths in an axonindependent manner. Although this is possible, one reason this may be unlikely is that commissural and sensory axons are juxtaposed within the same dorsal spinal cord tract (DLF), yet became myelinated at different rates and to different extents.

Beyond our study, other data further support the interpretation that axonal factors regulate early sheath growth and stabilization. For instance, tetanus-sensitive synaptic vesicle release regulates nascent sheath growth and stabilization, suggesting that axon-derived factors influence early sheath behaviors $[5,37,38]$. Neuronal activity and transmitter release may be integrated at the level of second messengers within nascent myelin sheaths, because specific $\mathrm{Ca}^{2+}$ patterns associate with sheath growth or retraction $[39,40]$. Notably, in these studies as well as our own work reported here, sheath behaviors were observed at early developmental stages immediately following initial axon wrapping. Axon- 
dependent regulation of sheath behavior would not be expected to occur at this stage if initial myelination were solely controlled by an oligodendrocyte-intrinsic program, and adaptive or axon-dependent control would instead emerge at a later developmental time point. Such activity-dependent and synaptic vesicle regulation at the early stages of sheath formation and growth suggest that experiences and adaptive changes to myelin can occur from the onset of myelination, calling question to the notion of separable and sequential hardwired then adaptive phases of myelination.

The subtype-specific differences in nascent sheath growth rate and pruning frequencies we observed may explain how some axons become myelinated more rapidly than others. For instance, the rapid growth rates and absence of sheath retractions associated with spinal cord commissural axons would predict rapid overall ensheathment, which is consistent with our observation that $t b \times 16^{+}$CoPA axons became completely myelinated within the $48 \mathrm{~h}$ after initial wrapping. Conversely, the comparatively slower sheath growth rates and increased pruning frequencies among nascent sheaths wrapping phox $2 b^{+}$reticulospinal axons corresponded to a reduced overall rate of myelination during the same time period. Based on available evidence from our study and others reporting that commissural and reticulospinal axons possess similar diameters at this developmental stage [25], these differential sheath behaviors are most likely not attributable to differences in axon caliber, and instead support the notion that axon-derived molecular signals are active regulators of sheath behavior during the earliest phases of myelination. These behaviors may explain the emergence of unique myelination patterns on distinct neuronal subtypes, such as those observed on axons positioned adjacent to one another within the mouse neocortex [36]. However, one limitation of this study is that myelin profiles of individual axons were not observed beyond late larval stages, and the extent to which larval axon ensheathment patterns are transient or sustained into later life stages in zebrafish are only beginning to be investigated [8].

\section{Can axons autonomously control their own myelination?}

The observed differences in the rates that individual axon subtypes become myelinated could be accomplished by one or more control mechanisms. First, if all myelin-competent axons are comparable targets for oligodendrocytes, then the rate that any individual axon becomes myelinated could be based on the local supply of oligodendrocytes and their limited myelinating capacity. Pointing against this possibility, we found that commissural and sensory axons become myelinated at vastly different rates, despite the fact that these axons both reside within the DLF. Furthermore, in the mouse cortex, myelination of distinct axon types initiates at different timepoints which would not be expected if myelination were solely determined by local supply of oligodendrocytes [36]. As an alternative to the oligodendrocyte supply model, the molecular properties of an individual axon could serve to define or instruct its own rate of myelination. In support, Auer et al. reported that following oligodendrocyte ablation, regenerated myelin tends to restore the same pattern and position of myelin that was found on individual axons prior to oligodendrocyte death [8]. Our axon ablation experiments also contribute to this question. We reasoned that if axons lacked the ability to dictate their own set point rate of myelination, they would become more rapidly and fully myelinated when the oligodendrocyte to target axon ratio was increased. Instead, we found that both spinal commissural and sensory axons maintained a constant rate of myelination, despite the surplus of oligodendrocytes relative to remaining target axons. Almeida et al. performed similar experiments but used a genetic approach rather than physical ablation to reduce the number of reticulospinal target axons within the posterior spinal cord. In this study oligodendrocytes did not increase myelination of RB sensory axons, but did occasionally perform ectopic myelination of cell bodies [27]. Inverse experiments that increased the target axon supply caused oligodendrocytes to increase myelinating potential in order to meet the increased demand imposed by supernumerary target axons, and ensure the normal rate of myelination for this reticulospinal (Mauthner axon) subtype [41]. Taken together, these studies indicate that some neuronal subtypes are hard-wired to instruct oligodendrocyte behavior during the initial stages of myelination, and further suggest that the mechanisms preventing ectopic or accelerated axon ensheathment may be dominant to those preventing myelination of cell bodies and dendrites.

\section{Implications for myelin plasticity and regeneration}

Oligodendrocytes and their progenitors detect and respond to changes in the local environment in various ways to ensure the intended distribution of myelin onto target axons. As recently discussed, increasing the number of target axons causes individual oligodendrocytes to increase their myelinating potential, but may not influence specification, proliferation, or differentiation of oligodendrocyte progenitor cells (OPCs) and oligodendrocytes [41]. In contrast, when the number of target axons is reduced, oligodendrocytes continue to differentiate but can be reduced in number due to apparent axon-dependent influences on OPC proliferation and survival $[32,42]$. How oligodendrocytes sense the number of target axons and relay this to the mitotic and apoptotic pathways is incompletely understood, but 
these mechanisms may exist to ensure that the proper amounts of myelin are formed at the right times and places during development, and may also enable adaptation to neuronal changes.

Our studies of how ensheathment of individual axons is influenced by changes to the availability of other target axons also has implications for myelin plasticity. Are the early phases of myelination mediated by hard-wired developmental processes that are controlled exclusively by gene regulatory networks intrinsic to neurons and oligodendrocytes? Alternatively, could experience-based changes modify gene regulatory networks and pathways controlling initial sheath growth, stabilization, and the extent and rate that individual axons become myelinated? These questions are incompletely resolved, but accumulating evidence indicate that multiple forms of distinct adaptions can occur, and that such changes may depend on developmental stage, anatomical region, and axon subtype identity $[6,25,43,44]$. Differences in neuronal excitability between neighboring axons may influence the extent that individual axons become myelinated $[5,25,38,45]$. Our observations that spinal commissural and sensory axons restrict additional myelin sheaths or more rapid ensheathment, even when myelinating potential is in excess, suggest that these subtypes would not invite or accept additional myelin if activity were altered in neighboring axons. Instead, our findings are consistent with the notion that any adaptive shifts inbetween axon subtypes occurring in response to altered activity or experience are likely to be highly restricted to specific subtypes, rather than widespread across all subtypes. For example, reticulospinal axons may permit surplus oligodendrocytes to form more, albeit shorter myelin sheaths along their length in an adaptive manner [27]. We did not observe this effect on CoPA and RB sensory axons in our study. Notably, this correlates with the participation of synaptic vesicle release in the myelination of reticulospinal axons, but not for CoPA axons [25].

If axons provide critical information that instructs early myelin sheath growth and stabilization, this suggests that strategies and regenerative therapies focused solely on manipulating oligodendrocyte biology may be inefficient or insufficient to induce remyelination. Instead, if the properties of demyelinated axons have changed, these axonal alterations may represent important targets in addition to promoting oligodendrocyte differentiation and myelinating potential. For example, emerging evidence indicates that axonal galectins mark nonmyelinated axon segments, and that galectins may be mis-expressed within Multiple Sclerosis lesions [46, 47]. Numerous additional negative regulators of myelination have been identified that if targeted, could mitigate the barriers to remyelination (reviewed by $[9,13,14]$ ). It will be interesting for future studies to identify spinal cord commissural axon properties responsible for the superior sheath stabilizing and growth inducing properties of this subtype in comparison to reticulospinal and spinal sensory axons. One possibility is that the levels of second messenger and kinase cascades within axons could account for such differences. For example, increased activation of Akt-mTOR signaling within cerebellar parallel fiber axons is sufficient to cause ectopic myelination of this normally unmyelinated population [21]. Because activating axonal pathways such as the Akt-mTOR pathway is also sufficient to increase axon diameter, such manipulations may be sufficient to induce both permissive and instructive axonal cues, and therefore may represent promising avenues for myelin regeneration.

\section{Conclusions}

This study provides insight into how individual axons obtain distinct myelin profiles during development, and how neuronal diversity contributes to early myelin patterns across the CNS. Our findings demonstrate that the oligodendrocyte intrinsic propensity to myelinate is overridden or modified by properties of individual axons in vivo. This axon-dependent regulation occurs from the onset of myelination. Taken together, our data support a model whereby axons are not passive substrates during initial myelination, but instead instruct oligodendrocyte behaviors to control early sheath growth, stabilization, and pruning.

\section{Supplementary information}

Supplementary information accompanies this paper at https://doi.org/10. 1186/s13064-020-00149-3.

Additional file 1: Figure S1. Characterization of transgenic reporter lines used. Figure S2. Ablation removes posterior reticulospinal axon segments without altering the number of COPA and RB local spinal cord axons. Figure S3. Effects of reticulospinal ablation on posterior spinal cord glia.

\section{Abbreviations \\ CNS: Central nervous system; CoPA: Commissural primary ascending; DLF: Dorsal longitudinal fasciculus; dpf: Days post-fertilization; hpf: Hours post-fertilization; hpi: Hours post-injury; nMLF: Nucleus of the medial longitudinal fasciculus; OPC: Oligodendrocyte progenitor cell; RB: Rohon- Beard; SRRF: Super-resolution radial fluctuations}

\section{Acknowledgements}

We thank Mary Halloran (University of Wisconsin) for the Tg(pitx2c:EGFP) line, Michael Lardelli (University of Adelaide) for the Tg(tbx16:EGFP) line, and Angie Ribera (University of Colorado - Anschutz Medical Campus) for the

Tg(is/1[ss]:Gal4-VP16, UAS:DsRed) line. We also thank John Henley and Craig Nelson (Mayo Clinic) for spinal cord transection assistance, Alexandria Hughes for assistance with SRRF, Erika Vail and Mary Diekmann for technical assistance, Kevin Okome and Nina Horabik for statistical consulting.

\section{Authors' contributions}

J.H.H., H.N.N., A.J.T., M.R.M., and A.J.K. designed research; all authors acquired and analyzed data; S.B., J.H.H. and H.N.N. performed statistical analyses; J.H.H. and H.N.N. wrote the manuscript, and all authors edited the manuscript. 


\section{Funding}

This work was supported by National Multiple Sclerosis research grant 5274A1/T (J.H.H.), NSF CAREER award IOS-1845603 (J.H.H.), Winona State Foundation Special Project grants 251.0225, 251.0253, and 251.0327 (J.H.H), Winona State Professional Improvement funds (J.H.H.), and Winona State student research supply grants (A.J.T., M.R.M., A.J.K., H.N.N., E.N.E., S.T.M.). No funding sources were involved in study design, data collection, data analysis, data interpretation, nor manuscript preparation.

\section{Availability of data and materials}

All datasets, zebrafish lines, plasmids, and other reagents are available upon request from the corresponding author.

\section{Ethics approval and consent to participate}

All animal work performed in this study was approved by and followed the guidelines of the Institutional Animal Care and Use Committee at Winona State University.

\section{Consent for publication}

Not applicable.

\section{Competing interests}

The authors declare that they have no competing interests.

Received: 29 August 2020 Accepted: 17 September 2020

Published online: 28 September 2020

\section{References}

1. Bercury KK, Macklin WB. Dynamics and mechanisms of CNS myelination. Dev Cell. 2015;32:447-58.

2. Sherman DL, Brophy PJ. Mechanisms of axon ensheathment and myelin growth. Nat Rev Neurosci. 2005;6:683-90.

3. Liu P, Du J-L, He C. Developmental pruning of early-stage myelin segments during CNS myelination in vivo. Cell Res. 2013;23:962-4.

4. Czopka T, Ffrench-Constant C, Lyons DA. Individual oligodendrocytes have only a few hours in which to generate new myelin sheaths in vivo. Dev Cell. 2013:25:599-609.

5. Hines JH, Ravanelli AM, Schwindt R, Scott EK, Appel B. Neuronal activity biases axon selection for myelination in vivo. Nat Neurosci. 2015;18:683-9.

6. Makinodan M, Rosen KM, Ito S, Corfas G. A critical period for social experience-dependent oligodendrocyte maturation and myelination. Science. 2012;337:1357-60.

7. Baumann N, Pham-Dinh D. Biology of oligodendrocyte and myelin in the mammalian central nervous system. Physiol Rev. 2001:81:871-927.

8. Auer F, Vagionitis S, Czopka T. Evidence for myelin sheath remodeling in the CNS revealed by in vivo imaging. Curr Biol. 2018;28:549-59.

9. Osso LA, Chan JR. Architecting the myelin landscape. Curr Opin Neurobiol. 2017:47:1-7.

10. Piaton G, Gould RM, Lubetzki C. Axon-oligodendrocyte interactions during developmental myelination, demyelination and repair. J Neurochem. 2010; 114:1243-60.

11. Snaidero N, Simons M. The logistics of myelin biogenesis in the central nervous system. Glia. 2017;65:1021-31.

12. Hughes EG, Appel B. The cell biology of CNS myelination. Curr Opin Neurobiol. 2016:39:93-100.

13. Klingseisen A, Lyons DA. Axonal regulation of central nervous system myelination: structure and function. Neuroscientist. 2018;24:7-21.

14. Almeida RG. The rules of attraction in central nervous system myelination. Front Cell Neurosci. 2018:12:367.

15. Simons M, Lyons DA. Axonal selection and myelin sheath generation in the central nervous system. Curr Opin Cell Biol. 2013;25:512-9.

16. Howe CL. Coated glass and vicryl microfibers as artificial axons. Cells Tissues Organs. 2006;183:180-94.

17. Lee S, Leach MK, Redmond SA, Chong SYC, Mellon SH, Tuck SJ, et al. A culture system to study oligodendrocyte myelination processes using engineered nanofibers. Nat Methods. 2012;9:917-22.

18. Rosenberg SS, Kelland EE, Tokar E, la Torre De AR, Chan JR. The geometric and spatial constraints of the microenvironment induce oligodendrocyte differentiation. Proc Natl Acad Sci U S A. 2008;105:14662-7.

19. Bullock PN, Rome LH. Glass micro-fibers: a model system for study of early events in myelination. J Neurosci Res. 1990;27:383-93.
20. Bechler ME, Swire M, Ffrench-Constant C. Intrinsic and adaptive myelinationa sequential mechanism for smart wiring in the brain. Dev Neurobiol. 2018; 78:68-79.

21. Goebbels S, Wieser GL, Pieper A, Spitzer S, Weege B, Yan K, et al. A neuronal $\mathrm{PI}(3,4,5) \mathrm{P3}$-dependent program of oligodendrocyte precursor recruitment and myelination. Nat Neurosci. 2017;20:10-5.

22. Mayoral SR, Etxeberria A, Shen Y-AA, Chan JR. Initiation of CNS myelination in the optic nerve is dependent on axon caliber. Cell Rep. 2018;25:544-50.

23. Gustafsson N, Culley S, Ashdown G, Owen DM, Pereira PM, Henriques R. Fast live-cell conventional fluorophore nanoscopy with ImageJ through superresolution radial fluctuations. Nat Commun. 2016;7:12471-9.

24. Hughes AN, Appel B. Oligodendrocytes express synaptic proteins that modulate myelin sheath formation. Nat Commun. 2019;10:4125-15.

25. Koudelka S, Voas MG, Almeida RG, Baraban M, Soetaert J, Meyer MP, et al. Individual neuronal subtypes exhibit diversity in CNS myelination mediated by synaptic vesicle release. Curr Biol. 2016;26:1447-55.

26. Wells S, Nornes S, Lardelli M. Transgenic Zebrafish recapitulating tbx16 gene Early developmental expression. PLoS One. 2011;6:e21559.

27. Almeida RG, Pan S, Cole KLH, Williamson JM, Early JJ, Czopka T, et al. Myelination of neuronal cell bodies when myelin supply exceeds axonal demand. Curr Biol. 2018:28:1296-305.

28. Wolman MA, Sittaramane VK, Essner JJ, Yost HJ, Chandrasekhar A, Halloran MC. Transient axonal glycoprotein-1 (TAG-1) and laminin-alpha1 regulate dynamic growth cone behaviors and initial axon direction in vivo. Neural Dev. 2008;3:6.

29. Thiele TR, Donovan JC, Baier H. Descending control of swim posture by a midbrain nucleus in zebrafish. Neuron. 2014;83:679-91.

30. Severi KE, Portugues R, Marques JC, O'Malley DM, Orger MB, Engert F. Neural control and modulation of swimming speed in the larval zebrafish. Neuron. 2014:83:692-707.

31. Sankrithi NS, O'Malley DM. Activation of a multisensory, multifunctional nucleus in the zebrafish midbrain during diverse locomotor behaviors. Neuroscience. 2010;166:970-93.

32. Almeida R, Lyons D. Oligodendrocyte development in the absence of their target axons in vivo. PLoS One. 2016;11:e0164432.

33. Espinosa-Hoyos D, Jagielska A, Homan KA, Du H, Busbee T, Anderson DG, et al. Engineered 3D-printed artificial axons. Sci Rep. 2018:8:478.

34. Bechler ME, Byrne L, Ffrench-Constant C. CNS myelin sheath lengths are an intrinsic property of Oligodendrocytes. Curr Biol. 2015:25:2411-6.

35. Redmond SA, Mei F, Eshed-Eisenbach Y, Osso LA, Leshkowitz D, Shen Y-AA, et al. Somatodendritic expression of JAM2 inhibits Oligodendrocyte myelination. Neuron. 2016;91:824-36.

36. Zonouzi M, Berger D, Jokhi V, Kedaigle A, Lichtman J, Arlotta P. Individual Oligodendrocytes show Bias for inhibitory axons in the Neocortex. Cell Rep. 2019;27:2799-808.

37. Mensch S, Baraban M, Almeida R, Czopka T, Ausborn J, Manira El A, et al Synaptic vesicle release regulates myelin sheath number of individual oligodendrocytes in vivo. Nat Neurosci. 2015;18:628-30.

38. Wake H, Ortiz FC, Woo DH, Lee PR, Angulo MC, Fields RD. Nonsynaptic junctions on myelinating glia promote preferential myelination of electrically active axons. Nat Commun. 2015;6:7844.

39. Krasnow AM, Ford MC, Valdivia LE, Wilson SW, Attwell D. Regulation of developing myelin sheath elongation by oligodendrocyte calcium transients in vivo. Nat Neurosci. 2018:21:24-8.

40. Baraban M, Koudelka S, Lyons DA. Ca 2+ activity signatures of myelin sheath formation and growth in vivo. Nat Neurosci. 2018;21:19-23.

41. Almeida RG, Czopka T, Ffrench-Constant C, Lyons DA. Individual axons regulate the myelinating potential of single oligodendrocytes in vivo. Development. 2011;138:4443-50.

42. Ueda H, Levine JM, Miller RH, Trapp BD. Rat optic nerve oligodendrocytes develop in the absence of viable retinal ganglion cell axons. J Cell Biol. 1999;146:1365-74.

43. Tomassy GS, Dershowitz LB, Arlotta P. Diversity matters: a revised guide to myelination. Trends Cell Biol. 2016;26:135-47.

44. Hughes EG, Orthmann-Murphy JL, Langseth AJ, Bergles DE. Myelin remodeling through experience-dependent oligodendrogenesis in the adult somatosensory cortex. Nat Neurosci. 2018;21:696-706.

45. Mitew S, Gobius I, Fenlon LR, McDougall SJ, Hawkes D, Xing YL, et al. Pharmacogenetic stimulation of neuronal activity increases myelination in an axon-specific manner. Nat Commun. 2018;9:306.

46. Díez-Revuelta N, Hiquero AM, Velasco S, Peñas-de-la-lglesia M, Gabius H-J, Abad-Rodríguez J. Neurons define non-myelinated axon segments by the 
regulation of galectin-4-containing axon membrane domains. Sci Rep. 2017;

7:12246.

47. de Jong CGHM, Stancic M, Pinxterhuis TH, van Horssen J, van Dam A-M, Gabius $\mathrm{H}-\mathrm{J}$, et al. Galectin-4, a negative regulator of Oligodendrocyte

differentiation, is persistently present in axons and microglia/macrophages in multiple sclerosis lesions. J Neuropathol Exp Neurol. 2018;77:1024-38.

\section{Publisher's Note}

Springer Nature remains neutral with regard to jurisdictional claims in published maps and institutional affiliations.

Ready to submit your research? Choose BMC and benefit from:

- fast, convenient online submission

- thorough peer review by experienced researchers in your field

- rapid publication on acceptance

- support for research data, including large and complex data types

- gold Open Access which fosters wider collaboration and increased citations

- maximum visibility for your research: over $100 \mathrm{M}$ website views per year

At $\mathrm{BMC}$, research is always in progress.

Learn more biomedcentral.com/submissions 